\title{
Criminal Registries, Community Notification, and Optimal Avoidance
}

\author{
Dan Simundza \\ University of Michigan
}

November 11, 2010

\begin{abstract}
This paper studies the effect of community notification of criminal registries on neighborhood behavior and shows that notification is not always optimal. I model notification as generating opposing externalities. Informed neighbors are harder to victimize without getting caught, which generates deterrence. But once informed, agents face private incentives to protect themselves through costly avoidance measures, thereby placing their neighbors at higher risk. My main results highlight the complementary relationship between government imposed penalties and notification policies. I prove that notification with too light of a penalty is bad for the community. Criminals cannot be deterred with light penalties despite the higher probability of detection; the informed therefore cower indoors and anyone venturing outside is an easy target. Conversely, there always exists a penalty severe enough to ensure that notification improves the community's welfare. With severe penalties, informed neighbors economize on costly avoidance strategies, further helping to deter crime and protect their community. Therefore, the government's decision to notify communities of criminals in their midst depends in part on how severely they want to penalize repeat offenders.
\end{abstract}




\section{Introduction}

Criminal registry notification laws provide information about offenders to at-risk neighbors with the intent of protecting the community. This paper investigates the effect of notification laws on the behavior of criminals and their law abiding neighbors and derives optimal notification policies. In my model, informed neighbors 1) can practice costly avoidance to protect themselves, and 2) better recognize and report criminal activity to the authorities, thereby increasing the probability of catching repeat offenders. Notification therefore generates opposing externalities; protecting oneself often comes at the cost of exposing one's neighbor, while increasing the probability of detection helps to deter criminal activity. Put simply, informed neighbors face a choice: remain outside in harm's way and help to deter crime, or retreat to the relative safety of their house at a cost.

Modeling the neighborhood as a game in which each informed family independently chooses its avoidance level (i.e. fraction of the day to stay indoors), I first study how notification policies affect neighborhood behavior. I show that avoidance obeys strategic complementarities: the more the neighbors stay inside, the higher the incentives for each family to remain indoors, possibly leading to a situation of hyper-avoidance. I prove that equilibria exist and are necessarily symmetric. Equilibrium avoidance may increase or decrease in the notification rate depending on whether informing the marginal citizen mainly serves to deter crime or decrease the average amount of time spent outside. I also show that equilibrium avoidance is a decreasing function of the penalty imposed by the government.

With a better understanding of the neighborhood's response, I turn to the issue of designing optimal notification policies. The government controls both the penalty on convicted felons and the notification rate, and the main results of the paper highlight the importance of getting these two policy levers working together. ${ }^{1}$ I show that there always exists a penalty large enough to ensure that equilibrium avoidance decreases in the fraction of the population informed. Whenever this is the case, social welfare is necessarily increasing in the notification rate, and therefore maximized by a "scarlet letter" policy which informs the entire neighborhood. The higher notification rate leads to

\footnotetext{
${ }^{1}$ The penalty could take many forms but all that matters in the analysis is the criminal's loss in utility from punishment. For instance, a fine of $\$ 1,000$ may reduce a criminal's utility by the same amount as a year in prison. These penalties would be equivalent in the model. The model is not dynamic so incarceration is not an issue.
} 
lower per-family expenditures on avoidance and higher deterrence, since the probability of detection is larger when more informed people are outside.

But this sword cuts both ways because notification with too small of a penalty is worse than useless: it is harmful. The informed alter their behavior at non-negligible costs and impose negative externalities on their neighbors. In this case, notification entails a cost but no benefit: the criminal is insufficiently deterred while residents suffer from staying indoors. If the penalty is too small, the government is better off keeping the criminals' identities secret. By not releasing any information the government ensures society does not waste energy on costly avoidance.

\section{$1.1 \quad$ Background}

The most prevalent type of criminal registry notification policy is "Megan's law," which requires states to notify the public of registered sex offenders in their neighborhood. ${ }^{2}$ But there are registries and notification policies for many other types of criminals. Some examples of approved or proposed registries and notification policies are for criminals convicted of elderly abuse, animal abuse, hate crimes, gang crimes and drug dealing [Welch [2008]]. Part of the rationale for community notification is that these types of offenders are especially prone to recidivism and often commit their crimes close to home. ${ }^{3}$ Notification is thought to help at-risk neighbors protect themselves by monitoring and avoiding the potential threats in their community. If the community is made aware of criminals' presence, they can take actions to protect themselves.

In practice governments do not directly choose what fraction of the neighborhood to inform. But they do choose the method and intensity of notification, which together determine to a large extent the fraction informed. Popular methods of notification include online databases searchable by anyone with an internet connection, individual letters sent in the post, holding a book at the police station which can be accessed by those deemed "at risk", and door-to-door notification. ${ }^{4}$ Each of these methods entails different costs.

\footnotetext{
${ }^{2}$ All 50 states currently have versions of Megan's law in place.

${ }^{3}$ Hanson et al. [2003] report that recidivism rates amongst sex offenders are at least $14 \%$ after 5 years, $20 \%$ after 10 years, and $30-40 \%$ after 20 years (these are understatements because some crimes go undetected). While criminal behavior for sex offenders declines in age of the offender, it does so more slowly than for other crimes (Hanson [2002]).

${ }^{4}$ Surveys described in Philips [1998], Kernsmith et al. [2009], and Lieb and Nunlist
} 
I ignore the physical costs of notification in this paper and instead focus on the costs generated by the strategic interaction of the informed neighbors. My goal is to clarify our understanding of the costly behavioral response to notification.

The reasoning supporting notification is that informed neighbors can better avoid potential threats, or at least limit their exposure. But as this paper shows, information can be a burden to the community. Informed families alter their behavior in order to reduce their chances of being victimized. Once a family learns there is a potential danger, it can no longer be as carefree: doors must remain locked, children must be supervised, and outdoor activity in general is curtailed. Such avoidance behaviors are costly. Along with the administrative costs and potential harm to the criminal, the cost of the neighborhood response should be a consideration in any cost-benefit analysis for notification laws.

I model avoidance as displacing crime. When $i$ stays inside more often, the probability $j$ is attacked increases because criminals are less likely to select $i$ from the crowd. The actual mechanism might be more subtle, but the results are the same. With the informed inside more often, there are simply less potential witnesses on the street, making crimes easier to commit. Further, when the informed are inside they cannot warn their neighbors and protect them as dangerous situations arise. If, in contrast, the informed stay outside more often, deterrence increases because they know who the criminals are and can watch over their neighbors. Criminals will be caught more often and will therefore be deterred from attacking unless the opportunity is especially good.

\subsection{Literature Review}

Since Becker [1968] the study of how criminals respond to incentives has flourished. Recent studies have investigated the deterrence effects of police and incarceration [Levitt [1997], Di Tella and Schargrodsky [2004]], conditions in prisons [Katz et al. [2003]], and gun ownership [Lott [1998], Mialon and Wiseman [2006]]. Other papers study the relationship between reputation concerns and violent crimes [Silverman [2004]], incarceration's effect on the supply of crime [Freeman [1996]], the interplay of crime and vigilance in a general equilibrium framework [Smith [2010]], and optimal law enforcement

[2008] provide information about notification methods and rates. 
[Eeckhout et al. [2010], Polinsky and Shavell [2000]]. My research adds to this literature by studying an additional crime fighting tool available to the government: community notification policies.

Previous research on criminal registries and notification policies has focused on sex offenders. These studies were primarily concerned with the effect notification had on recidivism rates and offender well-being [Prescott and Rockoff [2010], Adkins et al. [2000], Walker et al. [2005], Agan [2008], Simundza [2010]]. Much of this literature has difficulty finding any effect of registration and notification on recidivism rates. Prescott and Rockoff [2010] and Simundza [2010] show that registration programs reduce crime by providing police with better information on offenders' whereabouts, but the effect of notification programs is ambiguous. Notification programs reduce first time offenses by effectively increasing the penalty, but may increase recidivism rates by lowering the return to non-criminal activities and thereby the incentives for good behavior.

In my model the crime rate increases in the notification rate only when equilibrium avoidance increases sufficiently quickly. In such a situation, higher notification rates lead to a higher fraction of uninformed people on the street, making crime easier and more attractive.

The extent of the literature's focus on neighborhood well-being is to quantify the effect a nearby offender has on property values [Linden and Rockoff [2008], Pope [2008]]. The results generally show a negative relationship between the existence of a sex offender and housing values, with the drop in housing prices ranging from $2.3 \%$ to $18 \%$. I take this as evidence that the law-abiding public cares about the registry. Avoidance is unobservable and difficult to verify but the correlation between house price and notification implies that individuals' response to being informed is non-negligible.

This paper also contributes to a large literature on the value of publicly disclosing information, of which Jin and Leslie [2003] and Dranove et al. [2003] are particularly relevant. Jin and Leslie [2003] studies the restaurant hygiene report card program instituted in Los Angeles and Dranove et al. [2003] studies the health care provider report card program instituted in New York. While more information to the consumer increases social welfare in Jin and Leslie [2003], Dranove et al. [2003] find the opposite due to providers' ability to "game the system".

My result that notification can be welfare increasing or decreasing combines elements of Jin and Leslie [2003] and Dranove et al. [2003] since informed neighbors' incentives may not be aligned with society's. Once in- 
formed, a family faces private incentives to protect itself by staying off the street. From society's point of view, this is exactly the wrong thing to do. Informed families should be outside as much as possible, thereby increasing the deterrence effect and helping the entire neighborhood. Whether notification or secrecy is optimal depends precisely on how much deterrence informing provides relative to how much families keep off the streets. The government imposed penalty is needed to generate sufficient deterrence so that incentives are aligned and notification improves welfare.

\section{Model}

\subsection{Motivation}

Before going into detail, in this section I provide an intuitive story to motivate the model and fix ideas. Consider a neighborhood consisting of a past offender (i.e. a potential threat) and neighbors A and B. Suppose, due to low effort and insufficient resources devoted to notification, only A becomes informed of the criminal's existence. Neighbor A responds by undertaking avoidance measures while $\mathrm{B}$ does not alter his behavior. This avoidance reduces A's probability of being attacked but is costly. If the offender wants to commit a crime, the probability that B is the one attacked therefore increases. In this way notification generates negative externalities.

The positive externality, deterrence, is generated by the following mechanism. When informed, A learns the offender's characteristics and prepares himself in the event of an attack. If $\mathrm{A}$ is attacked, he is more likely to identify the offender as the person he saw in the registry, whereas B might have trouble remembering the characteristics of his attacker. The criminal is more likely to be caught if he attacks an informed neighbor. The criminal knows the government's notification policy and computes the expected probability of detection. Since he cannot identify who is informed, the deterrence helps everyone in the neighborhood.

\section{$2.2 \quad$ Model}

The neighborhood has a mass of criminals, normalized to 1, and a mass $P>1$ of families. A fraction $\beta$ of the families are informed of the criminals' existence, while fraction $1-\beta$ are uninformed and have no idea criminals 
might be nearby. ${ }^{5}$ That mass $\beta P$ of the families are informed is common knowledge between the informed families and the criminals, but the criminals cannot identify who is informed.

Informed families choose avoidance level $a \in[0,1]$, where $a$ represents the fraction of the day they "lay low" inside, away from harm. Avoidance level $a$ costs $c(a)$, where $c:[0,1] \mapsto[0, \infty)$ is increasing and convex and satisfies $c(0)=c^{\prime}(0)=0$. Uninformed families make no choice. I normalize the harm of being attacked to one; if a mass $m$ of criminals attack $i$, his utility decreases by $m$.

Criminals attack when the expected benefits outweigh the expected costs. I assume the value criminal $j$ derives from attacking a family is $\theta_{j} \sim U[0,1]$. The realization of $\theta_{j}$ is known to the criminal before he decides whether to attack. Attacking criminals randomly choose one (and only one) family to attack. If he attacks and does not get caught his payoff is $\theta_{j}$. If he attacks and gets caught, his payoff is $\theta_{j}-\tau$, where $\tau$ is the penalty imposed by the government. If the criminal does not commit the crime, he gets nothing; he cannot target another family.

When the criminal attacks an uninformed family, he is caught with probability $d$, while attacking an informed family results in a detection rate of $D \geq d$. These values are known to the criminal and the informed families.

Family $i$ 's expected cost of attacks when all other informed families use avoidance level $a$ and a mass of $m$ criminals attack is

$$
m \frac{1-a_{i}}{P(\beta(1-a)+(1-\beta))}=m \frac{1-a_{i}}{P(1-\beta a)}
$$

Equation 1 shows that each family's expected harm from attacks is equal to the amount of time it spends outside relative to the total amount of time all neighborhood families spend outside, scaled by the mass of attacking criminals (and the cost of being attacked, which is one).

There is a potential problem when everyone is informed and staying inside all day because if family $i$ considers spending any part of the day outside, it will be attacked infinitely often. The following assumption guarantees there will always be some families outside.

A1 The maximum amount of avoidance is $\bar{a}<1$.

\footnotetext{
${ }^{5}$ Later I will discuss the implications of relaxing this assumption and letting the uninformed do inference after observing a noisy signal of their neighbors' actions.
} 
In order to guarantee interior equilibria, I also make the following assumption on the cost curve.

A2 Staying inside all day is dominated by staying outside all day; that is: $c(\bar{a})>\frac{\bar{a}}{P(1-\bar{a})}$.

The qualitative results which follow do not depend on these two assumptions. Without them, the analysis would be a bit tedious, mainly due to worrying about corner solutions.

\subsection{Comments}

Since the neighborhood is composed of a continuum of agents, each individual's actions has negligible effect on the community. The analysis will focus on symmetric equilibria in which all informed agents take the same action. So even though each individual family's avoidance has negligible effect on the community, there is an externality generated by the avoidance of the informed as a group.

Avoidance generates strictly negative externalities in this paper. When A stays inside more often, not only does the conditional probability that $\mathrm{B}$ is attacked increase, but the level of deterrence decreases. This happens because $\mathrm{A}$ is informed and entails the higher detection rate $D$. When he stays inside more often, he is less likely to be randomly selected by the criminal, and so the criminal faces a lower expected probability of being caught. It is possible to imagine situations in which the action taken by the informed generates positive externalities. As an extreme example, suppose all informed families carry weapons at all times. This action would generate a positive externality for the uninformed because the criminal would worry that his potential victim is armed.

The literature on property values and notification laws reports significant results only for very small distances (i.e. less than 0.1 miles). Therefore, I think of the mass of the law-abiding neighbors relative to the mass of criminals as "small". It is difficult to explicitly define "small" in this context, but $P$ should be small enough to generate incentives for vigilance, and to make the externality effects relevant. 


\section{Non-Deterrable Criminals}

I start by analyzing non-deterrable criminals to isolate one aspect of the model. Without deterrence, notification generates purely negative externalities. Informed neighbors practice avoidance to protect themselves which shifts the expected cost of attacks onto their neighbors. This effect exists in the more general model with rational criminals, but is especially easy to see when criminals cannot be deterred by increases in the probability of detection.

The economics of crime literature has largely proceeded under the assumption that criminals respond to incentives. There is however evidence that some criminals cannot be deterred from committing crimes. ${ }^{6}$ What is important for this section is that non-deterrable criminals always commit crimes; any opportunity is sufficient for them. The analysis here will be brief with the intent of simplifying one aspect of the more complicated general model. $^{7}$

Informed family $i$ takes as given the other informed families' avoidance levels and chooses $a_{i}$ to minimize the expected costs of being attacked plus the costs of vigilance. That is, they solve:

$$
\max _{a_{i}}\left\{-\frac{1-a_{i}}{P(1-\beta a)}-c\left(a_{i}\right)\right\}
$$

Optimal avoidance is always interior because marginal benefits exceed marginal costs at $a_{i}=0$, and A2 ensures $a_{i}=\bar{a}$ is dominated by $a_{i}=0$. Optimal

\footnotetext{
${ }^{6}$ Lee and McCrary [2009] exploit the discontinuous increase in penalties as criminals turn 18 years old. They find an extremely small response: criminals a few days younger than 18 years old do not commit many more crimes than those a few days over 18 despite facing much shorter jail sentences. This, as they point out, could be evidence that the criminals are not responding to incentives, or simply myopia. Wright and Decker [1994] and Cromwell and Olson [2003] both study samples of burglars who have committed hundreds (and in some cases thousands) of thefts in their lifetimes. These criminals seem unable to resist committing a crime if the opportunity presents itself. That said, even these non-deterrable criminals regularly make "occupancy probes" in order to lower their probability of detection. An occupancy probes might take the form of consulting funeral announcements and/or contacting relevant houses by phone to make sure no one is home.

${ }^{7}$ Important details like the existence of an equilibrium will be ignored for now but proved for the more general model later in the paper.
} 
vigilance necessarily satisfies the first order condition

$$
\frac{1}{P(1-\beta a)}=c^{\prime}\left(a_{i}\right)
$$

The equation characterizing a symmetric equilibrium is

$$
\frac{1}{P(1-\beta a)}=c^{\prime}(a)
$$

From this equation we can deduce equilibrium behavior as the fraction of neighbors informed of the criminals' existence increases. When there is no possibility of deterrence, as the notification rate increases and more neighbors hide safely in their house, the amount of time each hides inside increases.

Lemma 1. For non-deterrable criminals, equilibrium avoidance increases in the notification rate.

Proof. As $\beta$ increases, the left hand side of (2) increases. In order to restore equality in the first order condition $a$ must increase.

Since criminals cannot be deterred from crime by the higher probability of detection, the only externality at play here is the negative externality of avoidance. A higher notification rate leads to more people who practice avoidance. With more people practicing avoidance, the marginal benefit of avoidance increases, which in turn causes everyone to practice yet more avoidance. Informed citizens face stronger incentives to stay inside, thereby increasing the expected harm to anyone who remains outside on the streets.

In this case, the government's optimal notification policy is to keep the criminals' identities secret. Define neighborhood welfare as the integral of the neighbor's utilities. Since notification entails costly avoidance but cannot deter crime, the government maximizes neighborhood welfare by informing no-one of the criminals' existence.

Lemma 2. For non-deterrable criminals, neighborhood welfare is decreasing in the notification rate. The optimal notification policy is to notify no one.

Proof. Write equilibrium avoidance as a function of the notification rate as $a(\beta)$. Since the mass of attacks is always one, neighborhood welfare as a function of the notification rate is

$$
w(\beta)=-1-P \beta c(a(\beta))
$$


As $\beta$ increases, both the fraction of the population bearing the cost of avoidance and the amount of avoidance they use increases. Welfare decreases in the notification rate and is therefore maximized at $\beta=0$.

Notification policies, in the absence of deterrence, are harmful at the societal level because they cause families to change their behavior (at a cost) but do not affect the mass of criminals who attack. Notification merely displaces crime from one group of citizens to another. The total amount of crime stays constant while expenditures on avoidance increase. The welfare maximizing policy is to notify no-one, and thus ensure society does not waste its effort on costly avoidance.

In order for notification to be good for the community, it therefore must be the case that criminals respond to incentives. The only way for the community to be made better off by notification is for the crime rate to decrease as more people learn of the criminals' existence. When more people know who is potentially dangerous, the criminals are more likely to get caught committing a crime. They therefore become more selective in determining when to attack, which decreases the danger to their neighbors.

\section{Rational Criminals}

I now turn to the more general model of rational criminals. The negative externality isolated in the analysis of non-deterrable criminals is still present, but it can be counteracted by the positive externality of deterrence. Criminals are more likely to be caught when attacking an informed neighbor, but he cannot identify who is informed and who is not. Holding everything else constant (including avoidance), higher notification rates therefore lead to more deterrence. Counteracting this is the fact that once informed, neighbors face a private incentive to practice avoidance which robs the neighborhood of the desirable deterrence effect.

Rational criminals commit crimes when the expected benefits outweigh the expected costs. Let $\delta$ represent the criminals' expected probability of detection, which will be determined by equilibrium behavior. The criminals attack when $(1-\delta) \theta+\delta(\theta-\tau) \geq 0$, or $\theta \geq \tau \delta$. Since criminal values are distributed uniformly on the unit interval, the mass of attacking criminals is $1-\tau \delta .^{8}$

\footnotetext{
${ }^{8}$ Of course, this is only true if $\tau \delta \in[0,1]$. I restrict attention to this case because when
} 
A symmetric equilibrium is defined by a cutoff criminal value $\underline{\theta}$ at which criminals are indifferent between attacking and not, and an avoidance level $a$ which is optimal for each informed family given that all other informed families use avoidance $a$. I will later show that any equilibrium is necessarily symmetric, so the analysis proceeds under this assumption.

Recall that the probability of being caught when attacking an uninformed family is $d$ and the probability when attacking an informed family is $D>d$. Criminals' expected probability of detection in an equilibrium where the notification rate is $\beta$ and all informed families use avoidance level $a$ is

$$
\delta(\beta, a)=\frac{(1-\beta) d+\beta(1-a) D}{1-\beta a}
$$

The cutoff criminal value is therefore

$$
\underline{\theta}=\tau \frac{(1-\beta) d+\beta(1-a) D}{1-\beta a}
$$

Family $i$ 's problem is to minimize the expected costs of attacks plus the cost of avoidance, taking other informed families' avoidance level $a$ and the cutoff criminal value described above as given:

$$
\max _{a_{i}}\left\{-\left(1-\tau \frac{(1-\beta) d+\beta(1-a) D}{1-\beta a}\right) \frac{1-a_{i}}{P(1-\beta a)}-c\left(a_{i}\right)\right\}
$$

Optimal avoidance is interior because marginal benefits exceed marginal costs at $a_{i}=0$, and A2 implies $a_{i}=\bar{a}$ is dominated by $a_{i}=0$. Family $i$ 's optimal avoidance necessarily satisfies the first order condition

$$
\left(1-\tau \frac{(1-\beta) d+\beta(1-a) D}{1-\beta a}\right) \frac{1}{P(1-\beta a)}=c^{\prime}\left(a_{i}\right)
$$

A symmetric equilibrium is characterized by the equation

$$
\left(1-\tau \frac{(1-\beta) d+\beta(1-a) D}{1-\beta a}\right) \frac{1}{P(1-\beta a)}=c^{\prime}(a)
$$

Note that avoidance obeys strategic complementarities. The expected harm of attacks for a family who remains outside increases as the neighbors spend more time indoors for the simple reason that there are less people on the $\tau \delta>1$, no crimes are committed. 
streets for the criminals to attack. Family $i$ 's incentives for avoidance are therefore increasing in the amount of avoidance their neighbors practice. This can be seen in family $i$ 's first order condition (3) because both terms on the left hand side are increasing in $a$, implying $a_{i}$ increases in $a$.

I start by studying the equilibrium behavior of criminals and neighbors with the goal of getting a better understanding of how equilibrium behavior depends on the notification rate. The following lemma shows that symmetric equilibria exist, and further, that all equilibria are necessarily symmetric. It does however allow for the possibility that multiple symmetric equilibria exist.

Lemma 3. Equilibria exist and are necessarily symmetric.

Proof. When $a=0$, the left hand side of (4) is strictly positive while the right hand side is zero. When $a=\bar{a}$, assumption A2 guarantees the right hand side is larger than the left hand side. The intermediate value theorem guarantees there is some $a \in(0, \bar{a})$ where (4) holds with equality.

Equilibria are necessarily symmetric because the marginal benefit of avoidance is independent of one's own avoidance while marginal costs are strictly increasing in own avoidance. It is impossible for marginal benefits to equal marginal costs at two or more distinct avoidance levels.

Multiple symmetric equilibria may exist depending on the cost function. Both the marginal benefit curve on the left hand side of (4) and the marginal cost curve are increasing in $a$. Without additional assumptions on the shape of marginal costs, the two curves could intersect multiple times. It is useful to note that, in any equilibrium, marginal costs intersect marginal benefits from below. If the marginal cost curve intersects the marginal benefit curve from above, then the point of intersection is a local minimum because increasing or decreasing $a$ would increase utility. This fact will be useful when evaluating comparative statics.

The comparative static I am most interested in is how equilibrium avoidance depends on the notification rate. The unambiguous result that equilibrium avoidance increases in the notification rate for non-deterrable criminals in Lemma 1 derives from the fact that the term $1 /(P(1-\beta a))$, the marginal benefit of avoidance when criminals are non-deterrable, increases in $\beta$ for all $a$. Rational criminals, however, can be deterred by notification; for fixed avoidance, the probability of detection increases in the notification rate. This 
means that the mass of attacking criminals, $1-\tau \delta(\beta, a)$, decreases in the notification rate. Whether equilibrium avoidance increases or decreases in the notification rate depends on how much extra deterrence the marginal informed citizen provides relative to how much less time the informed spend outside.

Before answering this question analytically, I will show graphically how the equilibrium adjusts as the notification rate increases. Equation 4 characterizes a symmetric equilibrium, equating marginal benefits to marginal costs. It is straightforward to show that the marginal benefit function obeys the single crossing property in $(a, \beta) .{ }^{9}$ The marginal benefit with a high notification rate crosses the marginal benefit with a low notification rate at most once and always from below.

This means there are two possible scenarios to consider as $\beta$ increases: 1 ) the marginal benefit curve is everywhere lower, and 2) the marginal benefit curve is lower for small $a$ and then larger for high levels of avoidance. These possibilities, along with a hypothetical marginal cost curve, are depicted in the graph below where both $\beta_{1}$ and $\beta_{1}^{\prime}$ are larger than $\beta_{0}$.

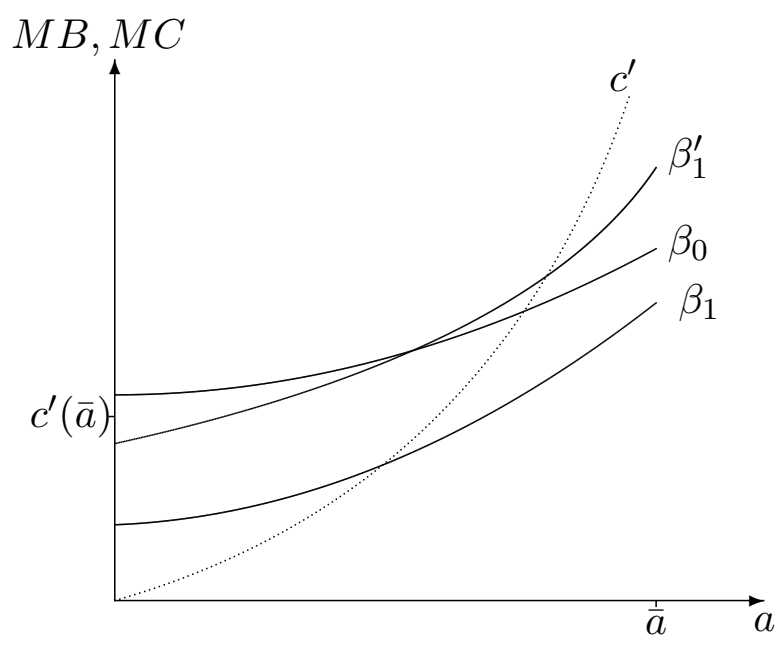

\footnotetext{
${ }^{9}$ This is done by differentiating$$
\left(1-\tau \frac{(1-\beta) d+\beta(1-a) D}{1-\beta a}\right) \frac{1}{P(1-\beta a)}
$$

with respect to $\beta$ and showing that if the derivative equals zero, the second derivative is always negative.
} 
From this graph we see that whether equilibrium avoidance decreases depends on how the two curves composing marginal benefits adjust as the notification rate increases. Avoidance decreases when $1-\tau \delta$ decreases more than $1 /(P(1-\beta a))$ increases at the original equilibrium. This can be seen by looking at what happens as $\beta_{0}$ increases to $\beta_{1}$. The marginal benefit curve is everywhere lower and so the intersection of marginal benefits and marginal costs occurs at a lower avoidance level.

On the other hand, if $1 /(P(1-\beta a))$ increases more than the mass of attacking criminals as the notification rate increases, equilibrium avoidance may increase. This can be seen by looking at what happens as $\beta_{0}$ increases to $\beta_{1}^{\prime}$. For the given cost function, the marginal benefit curve shifts up at the original equilibrium, and the new equilibrium entails higher avoidance.

The next result gives a more analytical description of what causes avoidance to increase or decrease as the notification rate changes. When the notification rate increases and avoidance is held constant, there are two effects that result from more people knowing who the criminals are. First, the criminals are deterred by the increased probability of getting caught. Second, the average time each family spends outside decreases, generating higher incentives for avoidance. Whether equilibrium avoidance increases or decreases depends on which of these effects dominates. If the deterrence effect dominates, then avoidance falls. If the strategic effect dominates, avoidance increases.

It is useful to write equilibrium avoidance as a function of the notification rate; that is, $a(\beta)$. When $\beta=0$, there is no avoidance because no one is informed of the criminals' existence. I define $a(0)=\lim _{\beta \rightarrow 0} a(\beta) .{ }^{10}$

Proposition 1. Equilibrium avoidance decreases in the notification rate if and only if, as the notification rate increases and avoidance is held constant, the effect on deterrence is sufficiently large relative to the effect on the average time spent outside per family.

Proof. Let $\pi(\beta, a) \equiv \frac{1}{P(1-\beta a)}$. Suppressing the arguments of $\delta(\beta, a)$ and $\pi(\beta, a)$ for notational simplicity, define the function

$$
h(a, \beta) \equiv(1-\tau \delta) \pi-c^{\prime}(a)
$$

\footnotetext{
${ }^{10}$ The function $a(\beta)$ is continuous at $\beta=0$ and the right-hand derivative exists. Equilibrium is defined as the intersection of the marginal cost curve with the marginal benefit curve. Marginal costs are constant in $\beta$, and marginal benefits are well behaved as $\beta \rightarrow 0$ : the marginal benefit approaches $(1-\tau d) / P$ for all $a$ as $\beta \rightarrow 0$. So $a(0)$ is simply the avoidance level where marginal costs equal $(1-\tau d) / P$.
} 
This function comes from the first order condition; in equilibrium, $h(a, \beta)=$ 0 . The implicit function theorem says that, since $h_{a}(a, \beta)<0$ in any equilibrium, the derivative $d a / d \beta$ exists and equals

$$
\frac{d a}{d \beta}=\frac{\tau \delta_{\beta} \pi-(1-\tau \delta) \pi_{\beta}}{(1-\tau \delta) \pi_{a}-\tau \delta_{a} \pi-c^{\prime \prime}(a)}
$$

The denominator is always negative because, in any equilibrium, marginal costs intersect marginal benefits from below ; otherwise the relevant $a$ would be a local minimum or point of inflection of utility. Avoidance decreases in $\beta$ when the numerator is positive, or

$$
\frac{\tau \delta_{\beta}}{1-\tau \delta}>\frac{\pi_{\beta}}{\pi}
$$

The right hand side of this inequality can be simplified to $\frac{a}{1-\beta a}$, so I re-write (6) as

$$
\frac{-\frac{\partial}{\partial \beta}[1-\tau \delta]}{1-\tau \delta}>\frac{-\frac{\partial}{\partial \beta}[1-\beta a]}{1-\beta a}
$$

The left hand side of this inequality is the percentage drop in the mass of attacking criminals. Since $1-\beta a=\beta(1-a)+(1-\beta)$, the right hand side is the percentage drop in the average amount of time each family spends outside.

This result helps to clarify equilibrium behavior as the notification rate increases. As more of the neighborhood learns of the criminals' existence, two things happen. First, there are more people who know to look out for the criminals and can better recognize them if an attack happens. This raises the probability an attacking criminal is caught and helps to deter crime. Second, there are more people who can now practice avoidance. If the deterrence effect of notifying additional neighbors is large enough, equilibrium avoidance decreases; everyone can spend a bit more time outside because the mass of criminals who have an incentive to attack decreases. If, on the other hand, notifying additional neighbors mainly serves to take them off the streets and increase the other families' probability of being targeted by criminals, then the equilibrium avoidance level will increase.

Building on the previous proposition, I derive a result on the shape of the equilibrium avoidance function. This corollary is primarily useful in proving 
the main results. Here I show that the equilibrium avoidance function is either always increasing, always decreasing, or single peaked in the notification rate.

Corollary 1. The equilibrium avoidance function is quasi-concave in the notification rate.

Proof. I show that whenever the slope of equilibrium avoidance given by (5) equals 0 , the second derivative is negative. The second derivative when $d a / d \beta=0$ is

$$
\frac{d^{2} a}{d \beta^{2}}=\frac{\left(\tau \delta_{\beta \beta} \pi+2 \tau \delta_{\beta} \pi_{\beta}-(1-\tau \delta) \pi_{\beta \beta}\right)\left((1-\tau \delta) \pi_{a}-\tau \delta_{a} \pi-c^{\prime \prime}(a)\right)}{\left((1-\tau \delta) \pi_{a}-\tau \delta_{a} \pi-c^{\prime \prime}(a)\right)^{2}},
$$

because $\tau \delta_{\beta} \pi-(1-\tau \delta) \pi_{\beta}=0$ by assumption. The denominator is always positive and $(1-\tau \delta) \pi_{a}-\tau \delta_{a} \pi-c^{\prime \prime}(a)<0$ because the marginal cost curve intersects the marginal benefit curve from below in any equilibrium. Simple algebra gives

$$
\tau \delta_{\beta \beta} \pi+2 \tau \delta_{\beta} \pi_{\beta}-(1-\tau \delta) \pi_{\beta \beta}=\tau \delta_{\beta} \frac{\delta_{\beta \beta}}{\delta_{\beta}} \pi-(1-\tau \delta) \pi_{\beta} \frac{\pi_{\beta \beta}}{\pi_{\beta}}+2 \tau \delta_{\beta} \pi_{\beta}
$$

Since

$$
\frac{\delta_{\beta \beta}}{\delta_{\beta}}=\frac{\pi_{\beta \beta}}{\pi_{\beta}}=\frac{2 a}{1-\beta a}>0,
$$

the expression in (7) becomes

$$
\frac{2 a}{1-\beta a}\left(\tau \delta_{\beta} \pi-(1-\tau \delta) \pi_{\beta}\right)+2 \tau \delta_{\beta} \pi_{\beta}
$$

Since $a^{\prime}(\beta)=0$ when $\tau \delta_{\beta} \pi-(1-\tau \delta) \pi_{\beta}=0$, second derivative $a^{\prime \prime}(\beta)<$ 0 whenever $a^{\prime}(\beta)=0$. This means $a^{\prime}(\beta)$ equals 0 at most once, and the equilibrium avoidance function is quasi-concave.

Corollary 1 implies that if equilibrium avoidance is decreasing at $\beta_{0}$, it is also decreasing for any $\beta_{1}>\beta_{0}$. Similarly, if equilibrium avoidance is increasing at $\beta_{1}$, it is increasing for any $\beta_{0}<\beta_{1}$. This result is useful because the behavior of equilibrium avoidance near the extreme values of $\beta$, which is often easiest to determine, can give information about the interior range. For instance, if $\lim _{\beta \rightarrow 0} a^{\prime}(\beta)<0$, then we know that equilibrium avoidance is decreasing in the notification rate at all possible notification rates. This fact will be used to prove the main results. 


\section{Welfare}

The analysis up to this point has been mostly positive in focus. Lemma 3, Proposition 1, and Corollary 1 help us to understand how the neighborhood responds to being notified an offender lives in their midst. In order to determine when notification is optimal a measure of community welfare is needed. I define welfare as the integral of the utilities of all of the neighbors living in the community, ignoring the criminals. ${ }^{11}$ Then equilibrium welfare is

$$
w(\beta)=-(1-\tau \delta(\beta, a(\beta)))-P \beta c(a(\beta))
$$

The next Proposition relates neighborhood welfare to equilibrium behavior when equilibrium avoidance is decreasing.

Proposition 2. Social welfare is increasing in the notification rate whenever equilibrium avoidance is decreasing in the notification rate.

Proof. Differentiating equilibrium social welfare with respect to the notification rate gives

$$
w^{\prime}(\beta)=\tau\left(\delta_{\beta}+\delta_{a} a^{\prime}(\beta)\right)-P c(a(\beta))-P \beta c^{\prime}(a(\beta)) a^{\prime}(\beta)
$$

The terms $\delta_{\beta}, \delta_{a} a^{\prime}$, and $-P \beta c^{\prime} a^{\prime}$ are positive, so it suffices to show $\tau \delta_{\beta}-$ $P c(a(\beta))>0$. I note three facts used to show this sufficient condition holds. Fact 1: since $a(\beta)$ is equilibrium avoidance, it gives higher utility than using $a=0$ :

$$
\begin{aligned}
& -(1-\tau \delta) \frac{1-a(\beta)}{P(1-\beta a(\beta))}-c(a(\beta))>-(1-\tau \delta) \frac{1}{P(1-\beta a(\beta))} \\
& \Rightarrow(1-\tau \delta) \frac{a(\beta)}{1-\beta a(\beta)}>\operatorname{Pc}(a(\beta))
\end{aligned}
$$

Fact 2: $a^{\prime}(\beta)<0 \Rightarrow \tau \delta_{\beta}>(1-\tau \delta) \frac{\pi_{\beta}}{\pi}$

Fact 3: $\frac{\pi_{\beta}}{\pi}=\frac{a(\beta)}{1-\beta a(\beta)}$.

The sufficient condition $\tau \delta_{\beta}-P c(a(\beta))>0$ holds because

$$
\tau \delta_{\beta}-P c(a(\beta))>\tau \delta_{\beta}-(1-\tau \delta) \frac{a}{1-\beta a}>(1-\tau \delta)\left(\frac{\pi_{\beta}}{\pi}-\frac{a(\beta)}{1-\beta a(\beta)}\right)=0,
$$

where the first inequality follows from Fact 1, the second from Fact 2, and the equality from Fact 3. I conclude that $a^{\prime}(\beta)<0 \Rightarrow w^{\prime}(\beta)>0$.

\footnotetext{
${ }^{11}$ Criminals only receive utility from successfully completing attacks. Including their utility in the welfare calculation would partially offset the lost utility of the neighbors.
} 
This result shows that when equilibrium avoidance decreases in the notification rate, informing more neighbors makes the community better off. Notifying more neighbors lowers the per-family expenditure on avoidance. This increases deterrence because more people know and those who know are outside more often. The proof shows that, despite increasing the mass of families who use avoidance (and therefore adjust their behavior at a cost), a marginal increase in the notification rate is welfare improving.

With these results in place, I now state and prove the main results of the paper. These results demonstrate the importance of the government imposed penalty in determining optimal notification policies. Specifically, penalties and notification complement one another. I first show that for severe enough penalties, equilibrium avoidance decreases over the entire range of notification rates and, as Proposition 2 says, neighborhood welfare therefore increases. This means that notification can be worthwhile for communities if the government imposes sufficiently severe penalties on repeat offenders.

Proposition 3. There always exists a severe enough penalty so that full notification is the optimal policy.

Proof. As the notification rate approaches zero, the slope of equilibrium avoidance from (5) is

$$
\lim _{\beta \rightarrow 0} a^{\prime}(\beta)=\frac{a(1-\tau d)-\tau(1-a)(D-d)}{P c^{\prime \prime}(a)},
$$

which is negative whenever

$$
\frac{a}{1-a}<\frac{\tau(D-d)}{1-\tau d}
$$

The right hand side of (8) increases in the penalty $\tau$ and gets arbitrary large as $\tau \rightarrow 1 / d$.

I now show that the left hand side of (8) approaches 0 for large enough penalties. Define $\kappa$ as inverse to marginal costs, so that $\kappa\left(c^{\prime}(a)\right)=a$. As $\beta \rightarrow 0$, equilibrium avoidance satisfies $\lim _{\beta \rightarrow 0} a(\beta)=\kappa((1-\tau d) / P)$. By the properties of the cost function, $\kappa$ is increasing and $\kappa(0)=0$. As $\tau \rightarrow 1 / d$, both avoidance and the function $a /(1-a)$ fall to 0 .

Then as $\tau \rightarrow 1 / d$, the left hand side of (8) approaches 0 while the right hand side gets arbitrarily large. This means for large enough $\tau, \lim _{\beta \rightarrow 0} a^{\prime}(\beta)<$ 0. By Corollary 1, equilibrium avoidance decreases over the entire range of 
notification rates and by Lemma 2 the neighborhood's welfare is maximized by notifying everyone.

Proposition 3 helps to understand both when and why community notification improves welfare. Severe penalties help the efficacy of notification. Large penalties on their own can deter crime, but the effect is magnified when coupled with community notification. The penalty and notification are complements. Informed neighbors entail a higher probability of detection, so the probability an attacking criminal has to pay the higher penalty increases with notification. This lowers the chance that a criminal will attack and also, importantly, the incentives for avoidance. The informed therefore spend more time outside, further generating deterrence.

The community is effectively empowered by public notification in conjunction with severe penalties. As more of the neighbors are informed, the probability of detection continues to rise and so the informed can spend more time outside instead of barricading themselves inside their house.

The next result provides a partial converse to Proposition 3 and is closely related to the analysis of non-deterrable criminals. If the penalty chosen by the government is too small, criminals will not be sufficiently deterred. In this case, informing generates a burden on society because the community spends a lot on avoiding criminals but cannot completely insulate itself. Someone is always left outside, providing an easy target for the attacking criminals.

Proposition 4. For small enough penalties, notifying no-one is optimal.

Proof. I will show that when the penalty is lenient enough, neighborhood welfare is decreasing for all $\beta$, implying the optimal notification rate is $\beta=0$. Equilibrium welfare is $w(\beta)=-(1-\tau \delta(\beta, a(\beta)))-P \beta c(a(\beta))$, and so the slope is

$$
w^{\prime}(\beta)=\tau\left(\delta_{\beta}+\delta_{a} a^{\prime}(\beta)\right)-P c(a(\beta))-P \beta c^{\prime}(a(\beta)) a^{\prime}(\beta)
$$

I first show that for small enough $\tau, a^{\prime}(1)>0$. Proposition 1 establishes that the slope of equilibrium avoidance is given by the Implicit Function Theorem. When $\beta=1$, it equals

$$
\left.\frac{d a}{d \beta}\right|_{\beta=1}=\frac{P \tau(D-d)-(1-\tau D) a(1)}{P^{2}(1-\tau D) \beta-P^{2}(1-a(1))^{2} c^{\prime \prime}(a(1))}
$$


The denominator is negative in any equilibrium, and for small enough $\tau$ the numerator is also negative, implying $a^{\prime}(1)>0$. Corollary 1 ensures that $a^{\prime}(\beta)>0$ for all $\beta$ whenever $\tau$ is small enough to make $a^{\prime}(1)>0$.

Then for $\tau$ small enough that $a^{\prime}(1)>0$, we know

$$
w^{\prime}(\beta)<\tau \delta_{\beta}-P c(a(\beta))
$$

because both $\delta_{a} a^{\prime}(\beta)$ and $-P \beta c^{\prime}(a(\beta)) a^{\prime}(\beta)$ are negative. The term $\delta_{\beta}$ is bounded above by $(D-d) /(1-\bar{a})^{2}$, so as $\tau \rightarrow 0$, we know $\tau \delta_{\beta} \rightarrow 0$.

This means we can choose $\tau$ small enough so that both $\tau \delta_{\beta}<\operatorname{Pc}(a(0))$ and $a^{\prime}(1)>0$ hold, guaranteeing that $w^{\prime}(\beta)<0$ for all $\beta$.

Very small penalties fail to deter crime, even if the probability of detection is high. In the extreme case where $\tau=0$, even certain detection cannot deter crime. Notification cannot generate sufficient deterrence with small penalties, despite the higher probability criminals are caught when attacking informed neighbors.

But notification imposes costs on the informed because they face high incentives to practice costly avoidance. From a welfare perspective, this avoidance is wasted because neighbors cannot isolate themselves from the criminals all of the time and crimes are committed. Notification has the effect of making the informed work harder without providing any benefit to the community.

As an extreme example, consider the limiting case of $\tau=0$. The model is then identical to the model with non-deterrable criminals where avoidance increases in the notification rate and welfare decreases in the notification rate. Informing no-one therefore maximizes welfare.

Criminal registry and community notification programs are sometimes thought of as a ways to keep offenders in "prison" even after they are released. Registered criminals are watched over more closely by local law enforcement and the community than if they were anonymous members of society. But this additional "prison sentence" is not enough; it cannot act as a substitute for a penalty. The community needs a sufficiently large penalty to use as a threat against the criminal in combination with a notification policy.

These results highlight the importance of getting the government's two policy levers working together. Before deciding whether to notify, the government needs to determine if the penalty is such that notifying will help or harm the community. 
The results in Propositions 3 and 4 result in zero or full-on notification as optimal policies. There are situations where the optimal notification policy is some interior fraction of the population. That is, informing some but not all of the neighborhood can maximize utilitarian social welfare. This occurs when then penalty is in some sort of "middle ground", neither severe nor lenient enough to generate unequivocal recommendations. This is why Proposition 4 is only a partial converse to Proposition 3.

\section{Discussion}

\subsection{Public Good vs. Public Bad}

The goal of this paper is to enhance our understanding of the effects of community notification on neighborhood behavior and welfare, and to determine when notification is optimal. How agents respond to being notified that an offender lives nearby is largely an empirical question. The action I study, avoidance, generates negative externalities. Here I discuss the possibility and implications of neighbors undertaking actions which generate positive externalities.

Data on how people alter their behavior in response to the threat of crime is scarce. I have not found studies which specifically deal with how neighbors change their behavior upon being notified a criminal lives nearby through community notification policies. In a national survey of 1,101 individuals, Ferraro [1995] asks a more general question: what activities have respondents undertaken to reduce their risk of crime? Their responses are summarized in Table 1.

Table 1 shows that people take actions which have both positive and negative externalities in order to protect themselves from the threat of crime. For instance, avoiding unsafe areas only serves to make those areas more unsafe for the people who must go there. Avoidance is thus a public bad. On the other hand, criminals are less likely to attack if they are aware that some potential victims know self defense or might be carrying a weapon. This type of vigilance therefore creates a public good. Which effect dominates is an empirical question I do not attempt to answer. It is worth pointing out that sometimes local governments pass laws limiting actions which generate positive externalities in this context. For instance, Washington D.C. had a ban on residents owning handguns from 1976-2008. 
Table 1: Actions Undertaken in Response to Fear of Crime and Their Likely Effect on the Community

\begin{tabular}{c|l|c} 
Externality & Action & $\%$ Yes \\
\hline \multirow{3}{*}{ Negative } & Avoid Unsafe Areas - Day & 51.7 \\
& Avoid Unsafe Areas - Night & 75.3 \\
& Additional Locks on House & 57.3 \\
& Buy Watchdog & 24.5 \\
\hline Positive \& Negative & Additional Outside Lighting & 57.5 \\
\hline \multirow{2}{*}{ Positive } & Learn Self Defense & 38.3 \\
& Keep Weapon in Home & 40.7 \\
& Carry Defensive Object & 18.6 \\
\hline
\end{tabular}

Source: Ferraro [1995]. Responses from a nationally representative survey of 1,101 individuals.

I will refer to any action which generates positive externalities for the neighborhood as "vigilance". The probability of a successful attack is lower against someone who is vigilant. Criminals are therefore less likely to attack people if they know that some are vigilant. As more people use vigilance, the deterrence effect increases.

If the actions people take entail positive as well as negative externalities, I expect notification to be optimal more often. That is, the results in this paper are possibly pessimistic towards notification policies. While the policy recommendation in this paper may be to not notify, a study which takes account of the positive externality of vigilance behaviors may recommend some positive amount of notification. Said differently, any time my results advise notification, a model which also takes account of vigilance efforts would also advise notification, but not necessarily the other way around.

\subsection{Inference by the Uninformed}

In this paper the uninformed are constrained to take no action. They are unaware that something bad could happen to them. An interesting extension to this model would allow the uninformed to conduct inference after observing a noisy signal which is correlated with the neighborhood's behavior. Uninformed people might look around while outside and notice there is 
no one else out. The lack of people outside might be because everyone else is indoors avoiding the potential threat, or simply for other irrelevant reasons (away on vacation, out to lunch, etc.).

The uninformed would draw inference from the signal and, based on their beliefs, determine their optimal amount of avoidance. An equilibrium of this extended model would be the avoidance level of the informed, and the avoidance level of the uninformed as a function of their signal such that each is optimal given their beliefs and their neighbors behavior.

The level of avoidance in the neighborhood increases when the uninformed are allowed to conduct inference and change their behavior. Obviously, their avoidance level will be higher because previously it was zero. But the avoidance level of the informed also increases because avoidance obeys strategic complementarities; the marginal benefit of avoidance increases the more one's neighbors practice avoidance.

\section{Conclusion}

This paper studies the effect of criminal registry notification policies on neighborhood behavior. Informed neighbors practice costly avoidance measures in order to protect themselves, which generates negative externalities for the community. The negative externality of avoidance can be counterbalanced by the positive externality of notification: notified neighbors are more difficult for the criminal to successfully victimize, generating deterrence effects for the entire community. Whether notification is optimal or not depends, in part, on the magnitude of these two effects.

The main results of this paper highlight the complementary nature of penalties and notification. In particular, Proposition 3 shows that there always exists a severe enough penalty to make notification optimal. The more people know of the criminals' existence, the more criminals are deterred and the safer it is for neighbors to remain outdoors. Conversely, Proposition 4 shows that when penalties are lenient the government maximizes welfare by not informing the community of the criminals' existence. Notification leads to costly expenditures on avoidance but insufficient deterrence; community notification cannot act as a substitute for lenient penalties.

In addition to the physical cost of informing the neighborhood, notification policies entail costs for communities because the informed change their behavior. Policy makers need to consider these behavioral costs of no- 
tification when conducting cost-benefit analyses of community notification programs.

\section{References}

Oliver D. Welch. Good enough for skinheads: How the proliferation of nonsexual criminal registries makes us less safe. 2008.

R. Karl Hanson, Kelly E. Morton, and Andrew J. R. Harris. Sexual offender recidivism risk: What we know and what we need to know. Annals of the New York Academy of Sciences, 989:154-166, Jun 2003.

R.K. Hanson. Recidivism and age: Follow-up data from 4,673 sexual offenders. Journal of Interpersonal Violence, 17(10):1046-1062, 2002.

Dretha M Philips. Community notification as viewed by washington's citizens (document 98-03-1101). Washington State Institute for Public Policy, Mar 1998.

Poco D. Kernsmith, Erin Comartin, Sarah W. Craun, and Roger M. Kernsmith. The relationship between sex offender registry utilization and awareness. Sexual Abuse, 21(2):181-193, 2009.

Roxanne Lieb and Corey Nunlist. Community notification as viewed by washington's citizens: A 10-year follow-up (document 08-03-1101). Washington State Institute for Public Policy, Mar 2008.

Gary Becker. Crime and punishment: An economic approach. Journal of Political Economy, 76:169-217, 1968.

Steven D. Levitt. Using electoral cycles in police hiring to estmate the effect of police on crime. American Economic Review, 87(3):270-290, 1997.

Rafael Di Tella and Ernesto Schargrodsky. Do police reduce crime? estimates using the allocation of police forces after a terrorist attack. American Economic Review, 94(1), 2004.

Lawrence F. Katz, Steven D. Levitt, and Ellen Shustorovich. Prison conditions, capital punishment and deterrence. American Law and Economics Review, 5(2):318-343, 2003. 
John Lott. More Guns, Less Crime. University of Chicago Press, Chicago, 1998.

Hugo M. Mialon and Thomas Wiseman. The impact of gun laws: A model of crime and self defense. Economics Letters, 88:170-175, 2006.

Dan Silverman. Street crime and street culture. International Economic Review, 45(3):761-786, 2004.

Richard B. Freeman. Why do so many young american men commit crimes and what might we do about it? Journal of Economic Perspectives, 10(1): 24-42, 1996.

Lones Smith. Crime and vigilance as a large game. Unpublished Notes, 2010.

Jan Eeckhout, Nicola Persico, and Petra Todd. A theory of optimal random crackdowns. American Economic Review, 100(3), 2010.

A. Mitchell Polinsky and Steven Shavell. The economic theory of public enforcement of law. Journal of Economic Literature, 38(1):45-76, 2000.

J.J. Prescott and Jonah E. Rockoff. Do sex offender registration and notification laws affect criminal behavior? Journal of Law and Economics, 53, 2010 .

G. Adkins, D. Huff, and P. Stageberg. The Iowa sex offender registry and recidivism. Report of the Iowa Department of Human Rights, Division of Criminal and Juvenile Justice Planning and Statistical Analysis Center, 2000 .

J.T. Walker, S. Maddan, B.E. Vàsquez, A.C. VanHouten, and G. ErvinMcLarty. The influence of sex offender registration and notification laws in the united states. Arkansas Crime Information Center Working Paper, 2005 .

Amanda Agan. Sex offender registries: Fear without function? SSRN Working Paper, 2008.

Dan Simundza. Optimal timing of community notification. Unpublished Manuscript, 2010. 
Leigh Linden and Jonah E. Rockoff. Estimates of the impact of crime risk on property values from megan's laws. American Economic Review, 98(3): 1103-1127, 2008.

Jaren C Pope. Fear of crime and housing prices: Household reactions to sex offender registries. Journal of Urban Economics, 64(3):601-614, 2008.

Ginger Jin and Phillip Leslie. The effects of information on product quality: Evidence from restaurant hygiene grade cards. Quarterly Journal of Economics, 118:409-451, 2003.

David Dranove, Mark Satterthwaite, David Kessler, and Mark McClellan. Is more information better? the effects of "report cards" on health care providers. Journal of Political Economy, pages 555-588, 2003.

David S. Lee and Justin McCrary. The deterrence effect of prison: Dynamic theory and evidence. Working Papers 1168, Princeton University, Department of Economics, Center for Economic Policy Studies, Jul 2009.

Richard Wright and S. Decker. Burglars on the Job: Streetlife and Residential Break-Ins. Northeastern University Press, Boston, 1994.

Paul F. Cromwell and James N. Olson. Breaking and Entering: Burglars on Burglary. Wadsworth Publishing Company, Address, 2003.

Kenneth F. Ferraro. Fear of Crime: Interpreting Victimization Risk. State University of New York Press, Albany, NY, 1995. 